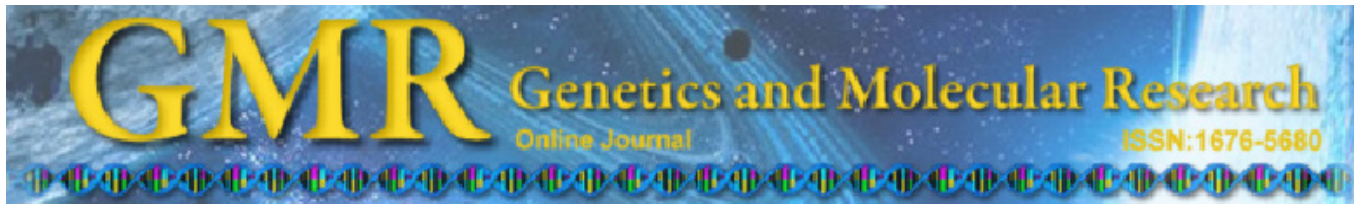

\title{
Aberrant DNA methylation of the P16, MGMT, and $h M L H 1$ genes in combination with the methylenetetrahydrofolate reductase C677T genetic polymorphism and folate intake in gastric cancer
}

\author{
J. Lin, R.M. Zeng, R.N. Li and W.H. Cao \\ China \\ Corresponding author: J. Lin \\ E-mail: linjing2999@aliyun.com / linjingst@tom.com
}

The First Affiliated Hospital of Shantou University Medical College, Shantou,

Genet. Mol. Res. 13 (1): 2060-2068 (2014)

Received April 11, 2013

Accepted October 31, 2013

Published March 24, 2014

DOI http://dx.doi.org/10.4238/2014.March.24.10

\begin{abstract}
Epidemiological studies have indicated that folate metabolism is correlated with increased risk of gastric cancer. Since methylenetetrahydrofolate reductase (MTHFR) is an important enzyme involved in folate metabolism, in this study, we examined whether polymorphisms and haplotypes of $M T H F R$ are correlated with the risk of gastric cancer. The polymorphisms MTHFR C677T and MTHFR A1298C were genotyped by polymerase chain reaction (PCR)-restriction fragment length polymorphism (RFLP) analysis in 285 patients and 570 healthy controls. Association analyses based on binary logistic regression were conducted to determine the odds ratio (OR) and its $95 \%$ confidence interval $(95 \% \mathrm{CI})$ for each genotype. The MTHFR 677TT genotype was significantly related with a reduced risk of gastric cancer $(\mathrm{OR}=0.60,95 \% \mathrm{CI}=0.39-0.92)$ compared to the $\mathrm{CC}$ genotype. Similarly, the MTHFR $1298 \mathrm{CC}$ genotype was significantly
\end{abstract}


associated with a decreased risk of cancer $(\mathrm{OR}=0.52,95 \% \mathrm{CI}=0.32$ 0.81). Haplotype analysis showed that the TC haplotype was associated with a reduced risk of gastric cancer compared to the most common haplotype, $\mathrm{CA}(\mathrm{OR}=0.28,95 \% \mathrm{CI}=0.12-0.60)$. Our results suggest that the MTHFR C677T and MTHFR A1298C polymorphisms are related to gastric cancer susceptibility in the Chinese population.

Key words: Methylenetetrahydrofolate reductase; Gastric cancer; Susceptibility; Haplotype analysis

\section{INTRODUCTION}

Gastric cancer is one of the most common types of tumors worldwide, and it ranks as the second leading cause of cancer-related deaths (Lao-Sirieix et al., 2010). Its incidence shows wide geographical variation, and almost two-thirds of all gastric cancer cases occur in developing countries, with $42 \%$ of them occurring in China alone. Based on the incidence and mortality rates of gastric cancer, the absolute number of cases is predicted to increase by the year 2050 (Forman and Burley, 2006). Gastric cancer is caused by multiple factors, including environmental and genetic factors, which may interact to affect tumor promotion and progression (Hamajima et al., 2006; Bai et al., 2008; Pan et al., 2012). Epidemiological studies have indicated that folate metabolism is correlated with an increased risk of gastric cancer, and that low intake of folate and polymorphisms in folate-metabolizing genes affect folate metabolism, and thus affect the susceptibility to gastric cancer (Choi and Mason, 2000; Miao et al., 2002; Götze et al., 2007).

Methylenetetrahydrofolate reductase (MTHFR), an important enzyme in folate metabolism, has a major impact on the regulation of the folic acid pathway due to the conversion of 5,10 methylenetetrahydrofolate (methylene-THF) to 5-methyl-THF. The most common polymorphisms in the MTHFR gene are C677T and A1298C, and consequent variations of the protein could change the enzyme activity. In particular, decreases in methyl-THF are associated with hyperhomocysteinemia, especially in a folate deficiency state. The MTHFR C677T (rs1801133) and MTHFR A1298C (rs1801131) variants have been associated with decreased MTHFR activity, increased homocysteine levels, and an altered mechanism of folate (Frosst et al., 1995; Bagley and Selhub, 1998; Brattström et al., 1998). The frequencies of MTHFR C677T (rs1801133) and MTHFR A1298C (rs1801131) vary across different ethnic groups, and variants of the two proteins commonly occur in Caucasian, Asian, and Hispanic populations, with a variation rate of 25 to $45 \%$ (Botto and Yang, 2000; Inoue et al., 2007). Previous experimental studies indicated that the low activity of MTHFR C677T (rs1801133) and MTHFR A1298C (rs1801131) are correlated with DNA hypomethylation, which may cause genomic instability and inactivate the tumor suppression genes (Brockton, 2006). Previous epidemiological studies have shown that variants of MTHFR might play an important role in the susceptibility to cancers and some disorders, such as colorectal cancer, cervical cancer, gastric cancer, vascular diseases, and neural tube defects (Neves et al., 2010; Teng et al., 2013; van Huis-Tanja et al., 2013; Yu et al., 2013). The association between gastric cancer risk and variants of MTHFR has been widely investigated; however, the results are inconsistent (Cui et al., 2010; Dong et al., 2010; Neves et al., 2010). Small sample sizes; inclusion of various ethnic groups; and variations in diet, environmental factors, and study methods 
may contribute to the discrepancies across these studies. We conducted a case-control study to determine whether polymorphisms and haplotypes of MTHFR are correlated with the risk of gastric cancer.

The P16, MGMT, and HMLH1 genes are important tumor suppression and DNA repair genes, and previous studies have shown that inactivation of these genes could increase the carcinogenesis of various cancers, including esophageal cancer. However, there is limited evidence about the role of the aberrant hypermethylation of these cancer-related genes ( $P 16$, $M G M T$, and $H M L H 1$ ) in the Chinese population. Here, we report the results of a case-control study that aimed to explore the association of MTHFR genetic polymorphisms to cancer and the relationship of P16, MGMT, and HMLH1 to MTHFR.

\section{MATERIAL AND METHODS}

\section{Subjects}

A total of 325 patients with gastric cancer confirmed by pathological diagnosis were recruited in The First Affiliated Hospital of Shantou University between January 2008 and December 2011. The age of patients ranged from 35 to 77 years. The exclusion criteria were patients with cardiac adenocarcinoma, secondary or recurrent tumors, a history of other malignant neoplasms, and previous eradication therapy for Helicobacter pylori infection. Ultimately, 285 patients were included in the final statistical analysis, with a participation rate of $87.7 \%$.

The control group comprised 570 subjects who were randomly selected from individuals who underwent health examinations during the same period with no history of cancer or other digestive system disease. All controls were frequency-matched by age (within 5 years) and gender with cases. The present study was approved by the Hospital Ethics Committee of the First Affiliated Hospital of Shantou University Medical College in China. All patients signed informed consent forms.

\section{Data extraction and quantification}

The questionnaire data and blood samples were obtained upon initial recruitment of cases and controls. Information about gender, age, and family history of cancer, tobacco usage, and smoking were collected from medical records by doctors or nurses.

Evidence of $H$. pylori infection was determined using the enzyme-linked immunosorbent assay (ELISA) for immunoglobulin $\mathrm{G}$ ( $\mathrm{IgG}$ ) antibodies (HpIgG ELISA) with a commercially available kit (Genesis Diagnostics; Cambridgeshire, UK) on sera obtained from $5 \mathrm{~mL}$ blood according to manufacturer instructions.

\section{Determination of folate concentration}

Folate intake was estimated through a comprehensive quantitative food-frequency questionnaire (FFQ) designed by the US National Institutes of Health and modified according to common Chinese food items and cooking habits. The food questionnaire contained 95 single food items with quantity and frequencies in five categories. The daily intake of folate was calculated by multiplying the folate content of the food by the total food intake (in grams) 
reported by the subjects. We then summarized the total folate intake by summing the separate folate intakes from various foods. Subjects were divided into two groups depending on folate intake: low intake $(\leq 260 \mu \mathrm{g} /$ day $)$ and high intake $(>260 \mu \mathrm{g} /$ day $)$.

\section{Genotyping}

All participants were asked to provide $5 \mathrm{~mL}$ venous blood, and genomic DNA was extracted from the blood samples using the Qiagen Blood Kit (Qiagen; Chatsworth, CA, USA). The forward and reverse primers of MTHFR C677T and MTHFR A1298C were designed by using Premier 5.0 software (Table 1). Genotyping of MTHFR C677T and MTHFR A1298C were accomplished by polymerase chain reaction (PCR)-restriction fragment length polymorphism (RFLP) analysis. The PCR conditions were as follows: initial denaturation at $94^{\circ} \mathrm{C}$ for $2 \mathrm{~min}$, followed by 35 cycles at $94^{\circ} \mathrm{C}$ for $30 \mathrm{~s}$, annealing at $60^{\circ} \mathrm{C}$ for $65 \mathrm{~s}$, extension at $72^{\circ} \mathrm{C}$ for $90 \mathrm{~s}$, and a final extension at $72^{\circ} \mathrm{C}$ for $5 \mathrm{~min}$. A random sample of $5 \%$ of cases and controls was genotyped again by different researchers. The reproducibility was $100 \%$.

\begin{tabular}{|c|c|c|c|c|}
\hline MTHFR & & Primers & Fragment length (bp) & Annealing temperature $\left({ }^{\circ} \mathrm{C}\right)$ \\
\hline$\overline{\mathrm{C} 677 \mathrm{~T}}$ & $\begin{array}{l}\text { Forward } \\
\text { Reverse }\end{array}$ & $\begin{array}{l}\text { 5'-CGTGGCTCCTGCGTTTCC-3' } \\
\text { 5'-GAGCCGGCCACAGGCAT-3' }\end{array}$ & 387 & 56 \\
\hline A1298C & $\begin{array}{l}\text { Forward } \\
\text { Reverse }\end{array}$ & $\begin{array}{l}\text { 5'-CAAATCTGAGGGAGCTGAGT-3' } \\
\text { 5'-CAGATAAGTGGCAGTACAGA-3' }\end{array}$ & 168 & 55 \\
\hline
\end{tabular}

\section{Statistical analysis}

All analyses were performed with the SPSS version 13.0 statistical software (SPSS; Chicago, IL, USA). Demographic characteristics were compared between cases and controls by means of a chi-squared test. The distributions of MTHFR C677T (rs1801133) and MTHFR A1298C (rs1801131) genotypes between cases and controls were compared by the chisquared test, and the association between the MTHFR polymorphisms and gastric cancer risk was estimated by unconditional logistic regression analysis. The odds ratios (ORs) and $95 \%$ confidence intervals (CIs) were calculated by using a conditional logistic regression model that adjusted for potential risk factors. Subjects with the wild-type genotype were considered as the reference group. Deviations from Hardy-Weinberg equilibrium (HWE) were checked for in the control group with the chi-squared test. All comparisons were two-sided, and $\mathrm{P}<$ 0.05 was considered to be statistically significant.

\section{RESULTS}

The characteristics of the study population are presented in Table 2. A total of 285 cases and 570 controls were included in our analysis. We did not find significant differences between cases and controls in the distributions of age and gender, which suggested adequate frequency matching. We found that patients were more likely to have a smoking habit compared to controls, but there was no significant difference in drinking status between cases and controls. 


\begin{tabular}{|c|c|c|c|c|c|c|}
\hline & Cases & $\%$ & Controls & $\%$ & $\chi^{2}$ & $\mathrm{P}$ \\
\hline & $\overline{\mathrm{N}=285}$ & & $\overline{\mathrm{N}=570}$ & & & \\
\hline \multicolumn{7}{|l|}{ Age (years) } \\
\hline Mean age & $57.3 \pm 5.6$ & & $58.7 \pm 6.1$ & & & \\
\hline$<55$ & 118 & 41.5 & 249 & 43.6 & & \\
\hline$\geq 55$ & 167 & 58.5 & 321 & 56.4 & 0.25 & 0.61 \\
\hline \multicolumn{7}{|l|}{ Gender } \\
\hline Male & 186 & 65.3 & 372 & 65.3 & & \\
\hline Female & 99 & 34.7 & 198 & 34.7 & - & - \\
\hline \multicolumn{7}{|c|}{ Tobacco smoking } \\
\hline Never & 161 & 56.5 & 386 & 67.8 & & \\
\hline Ever & 124 & 43.5 & 184 & 32.2 & 7.64 & $<0.05$ \\
\hline \multicolumn{7}{|c|}{ Alcohol drinking } \\
\hline Never & 166 & 58.2 & 356 & 62.4 & & \\
\hline Ever & 119 & 41.8 & 214 & 37.6 & 1.06 & 0.31 \\
\hline \multicolumn{7}{|c|}{ Family history of cancer } \\
\hline No & 27 & 9.6 & 20 & 3.5 & & \\
\hline Yes & 258 & 90.4 & 550 & 96.5 & 13.01 & $<0.001$ \\
\hline \multicolumn{7}{|c|}{ Folate intake $(\mu \mathrm{g} / \mathrm{d})$} \\
\hline$\leq 260$ & 182 & 63.8 & 286 & 50.2 & & \\
\hline$>260$ & 103 & 36.2 & 284 & 49.8 & 10.9 & $<0.05$ \\
\hline \multicolumn{7}{|c|}{ H. pylori infection } \\
\hline Positive & 115 & 40.3 & 299 & 52.5 & & \\
\hline Negative & 170 & 59.7 & 271 & 47.5 & 11.15 & $<0.001$ \\
\hline
\end{tabular}

Table 3 shows the genotype distributions for MTHFR and their corresponding ORs and $95 \% \mathrm{CIs}$ for gastric cancer risk. The distributions of the MTHFR C677T and MTHFR A1298C polymorphisms in the controls conformed to HWE $(\mathrm{P}>0.05)$. The frequencies of the MTHFR $677 \mathrm{CC}$, CT, and TT genotypes were 51.4, 33.9, and $14.7 \%$ in patients with gastric cancer, and $43.5,35.7$, and $20.8 \%$ in healthy controls, respectively. The frequencies of the MTHFR 1298AA, AC, and CC genotypes were 47.6, 40.3, and $12.1 \%$ in patients with gastric cancer, and $39.5,41.2$, and $19.3 \%$ in healthy controls, respectively. We found a significant difference in the frequencies of the MTHFR C677T and MTHFR A1298C polymorphisms between groups. The frequencies of the MTHFR $677 \mathrm{~T}$ allele and $1298 \mathrm{C}$ allele were significantly lower in gastric cancer patients than in controls $(\mathrm{P}<0.05)$. Multiple logistic regression analysis showed that compared with the CC genotype, the MTHFR 677TT genotype was significantly related with a reduced risk of gastric cancer $(\mathrm{OR}=0.60,95 \% \mathrm{CI}=0.39-0.92, \mathrm{P}<$ 0.05 ) after adjusting for gender, age, tobacco smoking, family history of cancer, folate intake, and H. pylori infection. Similarly, compared with the 1298AA genotype, we found that the MTHFR $1298 \mathrm{CC}$ genotype was significantly associated with a decreased risk of cancer (OR $=0.52,95 \% \mathrm{CI}=0.32-0.81, \mathrm{P}<0.05)$, and that the $\mathrm{C}$ allele also showed a reduced risk $(\mathrm{OR}=$ $0.73,95 \% \mathrm{CI}=0.54-0.97, \mathrm{P}<0.05)$.

\section{Linkage disequilibrium (LD) and haplotype analysis}

We conducted haplotype analysis using the SHEsis software. The MTHFR C677T and MTHFR A1298C polymorphisms were in high LD $\left(\mathrm{D}^{\prime}=1.0\right)$ (Figure 1). Four main haplotypes of MTHFR were considered in the analysis of all subjects. The results of the individual-level haplotype analysis and gastric cancer risk are shown in Table 4. The CA haplotype was found to be the most common in both the case and control groups. We did not find significant differences in the distributions of haplotypes $\mathrm{CA}$ or TC between the two groups $(\mathrm{P}<0.05)$. 
Haplotype TC was associated with a reduced risk of gastric cancer compared to the most common haplotype, $\mathrm{CA}(\mathrm{OR}=0.58,95 \% \mathrm{CI}=0.39-0.89, \mathrm{P}<0.05)$.

Table 3. MTHFR C677T and MTHFR A $1298 \mathrm{C}$ genotype distributions and odds ratio for gastric cancer.
\begin{tabular}{lccccccc}
\hline MTHFR & Cases & $\%$ & Controls & $\%$ & $\chi^{2}$ & $\mathrm{P}$ & Adjusted OR $(95 \% \mathrm{CI})$ \\
\cline { 2 - 7 } & $\mathrm{N}=285$ & & $\mathrm{~N}=570$ & & & & \\
\hline C677T & 146 & 51.4 & 248 & 43.5 & & & \\
CC & 97 & 33.9 & 203 & 35.7 & & & $0.81(0.58-1.13)$ \\
CT & 42 & 14.7 & 119 & 20.8 & 6.4 & $<0.05$ & $0.60(0.39-0.92)$ \\
TT & 139 & 48.6 & 304 & 53.3 & & & $0.77(0.58-1.05)$ \\
T-allele & 136 & 47.6 & 225 & 39.5 & & & 1.0 \\
A1298C & 115 & 40.3 & 235 & 41.2 & & & $0.81(0.59-1.12)$ \\
AA & 34 & 12.1 & 110 & 19.3 & 9.22 & $<0.05$ & $0.52(0.32-0.81)$ \\
AC & 149 & 52.4 & 327 & 57.3 & & & $0.73(0.54-0.97)$ \\
CC & & & & & & & \\
C-allele & &
\end{tabular}

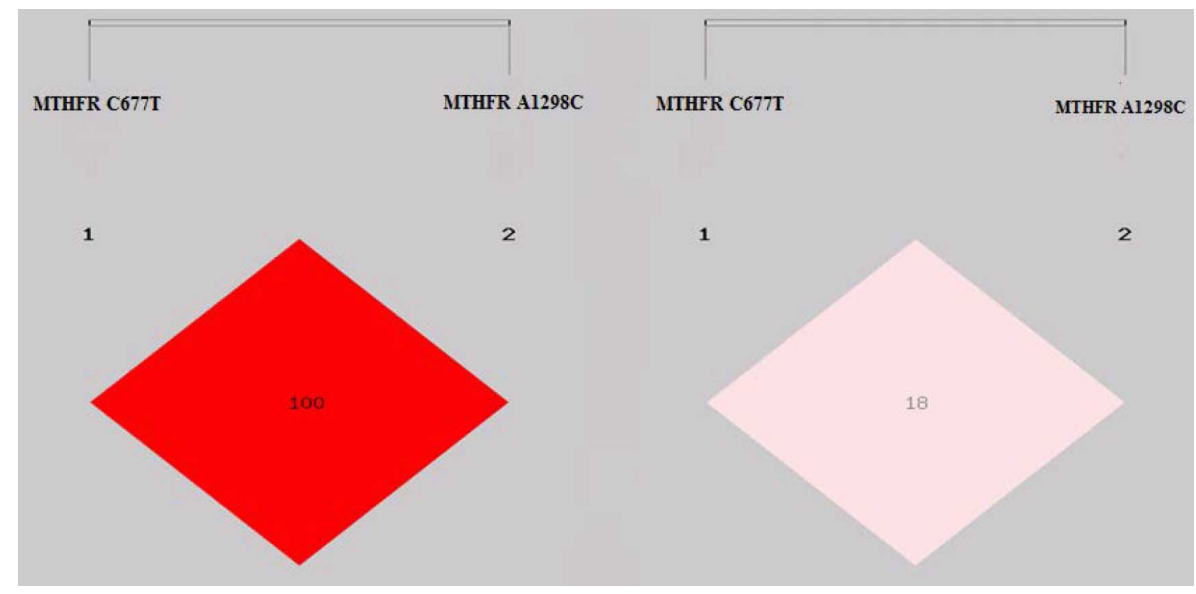

Figure 1. Linkage disequilibrium of the two SNPs by the SHEsis software.

Table 4. Association between haplotypes and gastric cancer for two SNPs in MTHFR.

\begin{tabular}{lccccccc}
\hline Haplotype & Cases & $\%$ & Controls & $\%$ & $\chi^{2}$ & P & Adjusted OR (95\%CI) \\
\hline CA & 134 & 47.1 & 219 & 40.5 & & & 1 \\
CC & 98 & 23.2 & 202 & 25.4 & 1.98 & 0.16 & $0.79(0.57-1.11)$ \\
TA & 53 & 33.7 & 149 & 27.7 & 2.68 & 0.11 & $0.58(0.39-0.89)$ \\
TC & 0 & 0 & 0 & 0 & NA & NA & NA \\
\hline
\end{tabular}

\section{DISCUSSION}

Gastric cancer is a complex disease that is caused by genetic and environmental factors and their interactions (Hamajima et al., 2006; Bai et al., 2008; Pan et al., 2012; Bastos et al., 2013). Increasing evidence indicates that the MTHFR C677T and MTHFR A1298C polymorphisms influence the risk of cancer (Sun et al., 2008; Tahara et al., 2009; Neves et 
al., 2010; Saberi et al., 2012). Folate mediates the transfer of one-carbon moieties during the process of DNA synthesis, replication, and repair, as well as DNA methylation reactions (Wang et al., 2008). MTHFR is a key enzyme in one-carbon metabolism, and the MTHFR variants could influence the levels of folate and homocysteine, and thus influence individual susceptibility to cancer.

In the present case-control study, we investigated the association between the MTHFR C677T and MTHFR A1298C polymorphisms and the risk of gastric cancer. Our results indicated that the frequencies of MTHFR C677T and MTHFR A1298C were significantly different between cases and controls. We found that the MTHFR 677TT and MTHFR 1298CC genotypes were associated with a reduced risk of gastric cancer, and that high folate consumption was correlated with a decreased risk of cancer. Moreover, haplotype analysis of the MTHFR C677T and A1298C polymorphisms showed that the TC haplotype was correlated with a reduced risk of gastric cancer when compared to the CA haplotype.

The association between the single-nucleotide polymorphisms (SNPs) in MTHFR and susceptibility to gastric cancer has been widely investigated, but the results are inconsistent (Sun et al., 2008; Dong et al., 2010). Several studies conducted in Chinese, Caucasian, and Mexican populations have shown that the MTHFR C677T polymorphism does not influence the risk of gastric cancer (Weng et al., 2006; Götze et al., 2007; Zhang et al., 2007). However, several studies conducted in Korean, Chinese, and Caucasian populations indicated the MTHFR 677TT genotype has a protective effect on the risk of gastric cancer. A meta-analysis of 22 studies including 4070 cases and 6462 controls suggested the MTHFR 677TT genotype influences susceptibility to gastric cancer, but no association was found between the MTHFR A1298C polymorphism and gastric cancer risk (Dong et al., 2010). By contrast, several studies observed positive associations between the MTHFR 677TT and 1298CC genotypes and gastric cancer risk (Boccia et al., 2007; Vollset et al., 2007; Yoo et al., 2012). In our study, we found that MTHFR 677TT and $1298 \mathrm{CC}$ had a 0.6 -fold and 0.52-fold risk of gastric cancer, respectively. The discrepancies of these results may be caused by different population backgrounds, sample sizes, control selection criteria, and interactions with other environmental factors. Therefore, further confirmation of this association is greatly needed.

To the best of our knowledge, our study was the first to investigate the association between polymorphisms in MTHFR and gastric cancer risk based on haplotype analysis. We found that the MTHFR C677T and MTHFR A1298C were in high LD $\left(\mathrm{D}^{\prime}=0.956\right)$, suggesting that the two SNPs are located in the same haplotype region. The results of SHEsis analysis showed that CA was the most common haplotype. The TC haplotype was associated with a reduced risk of gastric cancer in the Chinese population. These results indicated that the two SNPs, MTHFR C677T (rs1801133) and MTHFR A1298C (rs1801131), may interact, and that both might influence the susceptibility to gastric cancer.

Three limitations of our study should be considered. First, the cases and controls were only selected from one hospital, and the sample may therefore lack representation of the general population. Second, gastric cancer is a complex disease that is caused by both genetic and environmental factors. Other genetic and environmental factors may interact with the MTHFR polymorphisms. Third, the sample size was relatively small in our study, and further studies using large sample sizes are warranted to verify these results.

In conclusion, the present case-control study in a Chinese population found a protective effect of the MTHFR C677T and A1298C variant genotypes for gastric cancer, and we also found that the TC haplotype was significantly associated with reduced risk of gastric cancer. 
Our results suggest the MTHFR C677T and A1298C variants could influence the susceptibility to gastric cancer, and provide important information for biomarker research, therapeutic, and preventative strategies by molecular methods for gastric cancer.

\section{Conflicts of interest}

The authors declare no conflict of interest.

\section{ACKNOWLEDGMENTS}

Research approved and supported by Li Ka Shing Foundation Hospice Service.

\section{REFERENCES}

Bagley PJ and Selhub J (1998). A common mutation in the methylenetetrahydrofolate reductase gene is associated with an accumulation of formylated tetrahydrofolates in red blood cells. Proc. Natl. Acad. Sci. U. S. A. 95: 13217-13220.

Bai XL, Sun LP, Liu J, Chen W, et al. (2008). Correlation of interleukin-10-1082G/a single nucleotide polymorphism to the risk of gastric cancer in north China: a case-control study. Ai Zheng. 27: 35-40.

Bastos J, Peleteiro B, Pinto H, Marinho A, et al. (2013). Prevalence, incidence and risk factors for Helicobacter pylori infection in a cohort of Portuguese adolescents (EpiTeen). Dig. Liver Dis. 45: 290-295.

Boccia S, Gianfagna F, Persiani R, La Greca A, et al. (2007). Methylenetetrahydrofolate reductase C677T and A1298C polymorphisms and susceptibility to gastric adenocarcinoma in an Italian population. Biomarkers 12: 635-644.

Botto LD and Yang Q (2000). 5,10-Methylenetetrahydrofolate reductase gene variants and congenital anomalies: a HuGE review. Am. J. Epidemiol. 151: 862-877.

Brattström L, Wilcken DE, Ohrvik J and Brudin L (1998). Common methylenetetrahydrofolate reductase gene mutation leads to hyperhomocysteinemia but not to vascular disease: the result of a meta-analysis. Circulation 98: 2520-2526.

Brockton NT (2006). Localized depletion: the key to colorectal cancer risk mediated by MTHFR genotype and folate? Cancer Causes Control. 17: 1005-1016.

Choi SW and Mason JB (2000). Folate and carcinogenesis: an integrated scheme. J. Nutr. 130: 129-132.

Cui LH, Shin MH, Kweon SS, Kim HN, et al. (2010). Methylenetetrahydrofolate reductase C677T polymorphism in patients with gastric and colorectal cancer in a Korean population. BMC Cancer 10: 236.

Dong X, Wu J, Liang P, Li J, et al. (2010). Methylenetetrahydrofolate reductase C677T and A1298C polymorphisms and gastric cancer: a meta-analysis. Arch. Med. Res. 41: 125-133.

Forman D and Burley VJ (2006). Gastric cancer: global pattern of the disease and an overview of environmental risk factors. Best. Pract. Res. Clin. Gastroenterol. 20: 633-649.

Frosst P, Blom HJ, Milos R, Goyette P, et al. (1995). A candidate genetic risk factor for vascular disease: a common mutation in methylenetetrahydrofolate reductase. Nat. Genet. 10: 111-113.

Götze T, Rocken C, Rohl FW, Wex T, et al. (2007). Gene polymorphisms of folate metabolizing enzymes and the risk of gastric cancer. Cancer Lett. 251: 228-236.

Hamajima N, Naito M, Kondo T and Goto Y (2006). Genetic factors involved in the development of Helicobacter pylorirelated gastric cancer. Cancer Sci. 97: 1129-1138.

Inoue S, Hashiguchi M, Chiyoda T, Sunami Y, et al. (2007). Pharmacogenetic study of methylenetetrahydrofolate reductase and thymidylate synthase in Japanese and assessment of ethnic and gender differences. Pharmacogenomics 8: 41-47.

Lao-Sirieix P, Caldas C and Fitzgerald RC (2010). Genetic predisposition to gastro-oesophageal cancer. Curr. Opin. Genet. Dev. 20: 210-217.

Miao X, Xing D, Tan W, Qi J, et al. (2002). Susceptibility to gastric cardia adenocarcinoma and genetic polymorphisms in methylenetetrahydrofolate reductase in an at-risk Chinese population. Cancer Epidemiol. Biomarkers Prev. 11: 1454-1458.

Neves Filho EH, Alves MK, Lima VP and Rabenhorst SH (2010). MTHFR C677T polymorphism and differential methylation status in gastric cancer: an association with Helicobacter pylori infection. Virchows Arch. 457: 627-633.

Pan XF, Xie Y, Loh M, Yang SJ, et al. (2012). Polymorphisms of XRCC1 and ADPRT genes and risk of noncardia gastric cancer in a Chinese population: a case-control study. Asian Pac. J. Cancer Prev. 13: 5637-5642. 
Saberi S, Zendehdel K, Jahangiri S, Talebkhan Y, et al. (2012). Impact of methylenetetrahydrofolate reductase C677T polymorphism on the risk of gastric cancer and its interaction with Helicobacter pylori infection. Iran Biomed. J. 16: $179-184$

Sun L, Sun YH, Wang B, Cao HY, et al. (2008). Methylenetetrahydrofolate reductase polymorphisms and susceptibility to gastric cancer in Chinese populations: a meta-analysis. Eur. J. Cancer Prev. 17: 446-452.

Tahara T, Shibata T, Nakamura M, Yamashita H, et al. (2009). MTHFR 677T carrier influences the methylation status of H. pylori-infected gastric mucosa in older subjects. Dig. Dis. Sci. 54: 2391-2398.

Teng Z, Wang L, Cai S, Yu P, et al. (2013). The 677C > T (rs1801133) polymorphism in the MTHFR gene contributes to colorectal cancer risk: a meta-analysis based on 71 research studies. PLoS One 8: e55332.

van Huis-Tanja LH, Gelderblom H, Punt CJ and Guchelaar HJ (2013). MTHFR polymorphisms and capecitabine-induced toxicity in patients with metastatic colorectal cancer. Pharmacogenet. Genomics 23: 208-218.

Vollset SE, Igland J, Jenab M, Fredriksen A, et al. (2007). The association of gastric cancer risk with plasma folate, cobalamin, and methylenetetrahydrofolate reductase polymorphisms in the European Prospective Investigation into Cancer and Nutrition. Cancer Epidemiol. Biomarkers Prev. 16: 2416-2424.

Wang J, Sasco AJ, Fu C, Xue H, et al. (2008). Aberrant DNA methylation of P16, MGMT, and hMLH1 genes in combination with MTHFR C677T genetic polymorphism in esophageal squamous cell carcinoma. Cancer Epidemiol. Biomarkers Prev. 17: 118-125.

Weng YR, Sun DF, Fang JY, Gu WQ, et al. (2006). Folate levels in mucosal tissue but not methylenetetrahydrofolate reductase polymorphisms are associated with gastric carcinogenesis. World J. Gastroenterol. 12: 7591-7597.

Yoo JY, Kim SY, Hwang JA, Hong SH, et al. (2012). Association study between folate pathway gene single nucleotide polymorphisms and gastric cancer in Koreans. Genomics Inform. 10: 184-193.

Yu L, Chang K, Han J, Deng S, et al. (2013). Association between Methylenetetrahydrofolate reductase C677T polymorphism and susceptibility to cervical cancer: a meta-analysis. PLoS One 8: e55835.

Zhang FF, Terry MB, Hou L, Chen J, et al. (2007). Genetic polymorphisms in folate metabolism and the risk of stomach cancer. Cancer Epidemiol. Biomarkers Prev. 16: 115-121. 